University of Warwick institutional repository: http://go.warwick.ac.uk/wrap This paper is made available online in accordance with publisher policies. Please scroll down to view the document itself. Please refer to the repository record for this item and our policy information available from the repository home page for further information.

To see the final version of this paper please visit the publisher's website. Access to the published version may require a subscription.

Author(s): M. T. Downton and M. P. Allen

Article Title: Computer simulation of liquid-crystal surface modification Year of publication: 2004

Link to published version: http://dx.doi.org/10.1209/epl/i2003-10046-x

Publisher statement: None 
Europhys. Lett., 65 (1), pp. 48-54 (2004)

DOI: $10.1209 / \mathrm{epl} / \mathrm{i} 2003-10046-\mathrm{x}$

\title{
Computer simulation of liquid-crystal surface modification
}

\author{
M. T. Downton ${ }^{1}$ and M. P. Allen ${ }^{2}$ \\ 1 Centre for Molecular Simulation, Swinburne University of Technology \\ P.O. Box 218, Hawthorn, Victoria 3122, Australia \\ 2 Centre for Scientific Computing, University of Warwick \\ Coventry CV4 7AL, UK
}

(received 11 August 2003; accepted 17 October 2003)

PACS. 61.30.Hn - Surface phenomena: alignment, anchoring, anchoring transitions, surfaceinduced layering, surface-induced ordering, wetting, prewetting transitions, and wetting transitions.

PACS. 61.20. Ja - Computer simulation of liquid structure.

PACS. 02.70.Uu - Applications of Monte Carlo methods.

\begin{abstract}
Metropolis Monte Carlo simulations are used to study the interplay between two different anchoring effects of spherocylinders on a modified surface consisting of hard walls onto which liquid-crystal molecules have been perpendicularly grafted. By varying both the length and grafting density of the surface molecules, a number of different and novel anchoring regimes are observed including: planar, homeotropic, tilted and decoupled planar.
\end{abstract}

Introduction. - Much of liquid-crystal science focuses on the anchoring properties of the liquid on solid substrates [1-5]. The interest comes both from the desire to generate anchorings and director profiles that are technologically useful, and from the complexity of the interactions that can be studied [6]. Many methods exist for changing the anchoring properties of a system [7]. In this paper, the focus is on modifying a surface that gives planar anchoring by grafting onto it short alkyl chains that promote homeotropic alignment, a system which has been studied experimentally [8]. We aim to investigate the interplay between competing anchoring behaviours of this kind by performing simulations of a highly simplified model system of rod-like hard particles.

The paper is organised as follows. In the following section we present the computational details and molecular models that we use to simulate the liquid crystal and the modified surface. Simulation results are presented consisting of density, director, order parameter and biaxiality profiles for the modified systems. Finally, concluding remarks are given.

Molecular model and simulation methods. - The molecular model employed here reduces the physical system to its minimal constituent parts, the aim being to allow changes in the director orientation to be monitored through careful modification of the surface. Although the system of experimental interest is thermotropic, we chose to use hard spherocylinders for both the liquid-crystal model and the surface chains. The spherocylinder model is characterised by the length $L$ of the cylindrical section between hemispherical caps, and the diameter $D$. The 
bulk phase diagram is determined by the elongation, $L / D$, and the packing fraction

$$
\eta \equiv \frac{N v_{\mathrm{sc}}}{V}, \quad \text { where } v_{\mathrm{sc}}=\frac{\pi}{6} D^{3}\left[1+\frac{3}{2} \frac{L}{D}\right]
$$

is the volume of a single spherocylinder, $N$ is the number of particles, and $V$ the system volume. For hard-particle models of this kind, the temperature plays a trivial role in setting the energy scale of thermodynamic properties. Thus, although this simplification rules out any investigation of temperature effects, the entropic consequences of molecular packing on the bulk and surface phase behaviour are fully taken into account. These are the features of interest to us here. The bulk phase diagram of this system is well understood, and in particular the ranges of stability of nematic and smectic liquid-crystal phases are known as functions of density and molecular elongation [9]. Throughout this work we used $L / D=10$ and $\eta=0.293$, well within the nematic-phase region [9].

The simulation system consisted of a slab geometry with periodic boundary conditions in the $x y$ plane and hard walls in the $z$-direction; the walls were defined to exclude the internal volume of the spherocylinders. The alkyl chains were represented by fixing $N_{\mathrm{ch}}$ of the spherocylinders in random positions perpendicular to the walls; the cylindrical sections of the spherocylinders were indented into the wall by specified amounts to allow the effect of grafted chain length to be studied. Thus, there are two parameters to be studied in the simulation: the protruding chain length, $L_{\mathrm{ch}}$ and the surface concentration or grafting density of chains, which is represented in dimensionless units as a coverage

$$
\sigma \equiv \frac{N_{\mathrm{ch}} \pi D^{2}}{4 A}
$$

where $A$ is the total surface area. We used three values of chain length: $L_{\mathrm{ch}} / L=\frac{1}{3}, \frac{2}{3}, 1$. For each $L_{\text {ch }}$ we studied five surface coverages: $\sigma=1.25 \%, 2.5 \%, 3.75 \%, 5 \%, 10 \%$. The total number of particles was $N=1100$ and the box dimensions $L_{x} / D=L_{y} / D=25, L_{z} / D=50$. For one case, $L_{\mathrm{ch}} / L=\frac{2}{3}, \sigma=5 \%$, a system-size check was carried out with $N=4400$, doubling the $x$ and $y$ dimensions. A reference simulation for $N=1100$, with no grafted chains, was also performed.

Initial configurations were created at low density in a system with the walls well separated, and were allowed to equilibrate in the isotropic phase. This was followed by compression along the $z$-axis to the desired nematic density. Four realizations of the random grafted chain configurations were generated for each system to ensure some sampling of surface conformations. In each case, the grafting points on the surface were selected randomly with uniform probability subject to the restriction of non-overlap.

A standard constant- $N V T$ Metropolis Monte Carlo algorithm was used throughout this work; trial moves consisted of a rotation and translation of a spherocylinder and a Monte Carlo step was considered to be one trial move per particle. The size of trial moves was adjusted to give an acceptance ratio of around $25-30 \%$ as a balance between computational expense and efficiency of exploring phase space [10]. For each configuration a simulation consisted of the following:

- Initial equilibration of isotropic density liquid (50000 steps).

- Compression to nematic phase (300000 steps).

- Between two and seven equilibration runs of at least 500000 steps, depending on surface grafting density.

- One statistics-gathering run of 500000 steps. 
The degree of order in a region $\mathcal{R}$ of the liquid crystal can be found by diagonalising the tensor

$$
Q_{\alpha \beta}=\frac{1}{\mathcal{N}} \sum_{i \in \mathcal{R}}\left\langle\frac{3}{2} l_{i \alpha} l_{i \beta}-\frac{1}{2} \delta_{\alpha \beta}\right\rangle, \quad \alpha, \beta=x, y, z .
$$

The sum is over spherocylinders $i$ whose centers lie within region $\mathcal{R}$, and $\mathcal{N}$ is the number of such particles. The orientation of each particle is specified by the unit vector $\boldsymbol{l}_{i}$. The angle brackets $\langle\cdots\rangle$ represent a simulation average. The three eigenvalues of eq. (3) may be expressed $S,-\frac{1}{2} S+\frac{1}{2} P,-\frac{1}{2} S-\frac{1}{2} P$, where $S$ is the local nematic order parameter, and its corresponding eigenvector is the nematic director, $\boldsymbol{n} ; P$ is the local biaxiality parameter.

In the following sections, the regions $\mathcal{R}$ are defined as slices of width $\delta z=0.25 D$ perpendicular to the $z$-direction, resulting in profiles of order $S(z)$, biaxiality $P(z)$, director $\boldsymbol{n}(z)$ and packing fraction $\eta(z)$, averaged over the transverse $x, y$ dimensions. Apart from regions where the order parameter $S$ is very low, and the director therefore undefined, only the director tilt angle $\theta(z) \equiv \arccos \left(\left|n_{z}\right|\right), 0 \leq \theta(z) \leq \pi / 2$ was found to vary with $z$. The value of the polar angle $\phi(z) \equiv \arctan \left(n_{y} / n_{x}\right)$ is arbitrary in this geometry. Therefore, just the director tilt angle $\theta(z)$ is reported below.

In all simulations it was observed that the two surfaces behaved equivalently, and that the central region consisted of unstrained nematic liquid crystal, i.e. there was no change in the director orientation far from the surfaces, and no transmission of torque from one surface to another. Therefore, we present the results as averages over both surfaces.

Only the mobile spherocylinders are included in averages, i.e. the grafted chains are excluded. For systems that had almost identical profiles only one is shown and to save space not all densities of surface chain studied are given figures in the text if they were broadly similar to those at another density; where this occurs, it is mentioned in the text.

Results. - Figure 1 contains profiles of the unmodified system, i.e. zero coverage $\sigma=0 \%$ of surface chains. The planar walls induce director orientation in the plane of the surface, $\theta=\pi / 2$. Apart from this, the wall effects are only felt within a few diameters of the surface. Some layering of molecules parallel to the surface can be seen from peaks in $\eta(z)$, and there is an associated slight increase in the order parameter $S(z)$. The wall also induces a small non-zero biaxiality $P(z)$ due to a reduction in the freedom of molecules to reorient out of the surface plane.

Figure 1 also shows profiles corresponding to non-zero coverage of the short chains with $L_{\mathrm{ch}}=\frac{1}{3} L$. In no case does the surface modification induce any change in the director profile, and the wall effects are confined to a region of order $L$. As the surface coverage increases (the cases $\sigma=3.75 \%$ and $10 \%$ are illustrated), there is a general reduction of packing fraction within the range of the grafted chains, while the layering parallel to the surface on the range of a few $D$ persists. The most dramatic effect is the increase in biaxiality within the whole graft region. This is presumably due to a combination of effects: the grafted rods cause in-plane disorder, broadening the orientational distribution in the plane, while the wall acts to restrict out-of-plane orientational fluctuations.

For low coverage, there is a slight peak in packing fraction at $z \approx L_{\mathrm{ch}}+D$, corresponding to a weak tendency for molecules to lie parallel to the surface just above the grafted chain ends. For a surface coverage of $\sigma=10 \%$, the jump in density at this point is quite significant: even though this surface coverage is still quite low, it is tempting to regard the chain ends as constituting a new surface, acting to decouple the bulk liquid crystal from the planar wall.

At low coverage $\sigma=1.25 \%$ and half of the configurations studied with $\sigma=2.50 \%$, the longer grafted rods $L_{\mathrm{ch}}=\frac{2}{3} L$ induce a slight lowering of packing fraction for $z \leq L_{\mathrm{ch}}+$ $D$, relative to the unmodified surface, but otherwise have little effect. However, increasing 


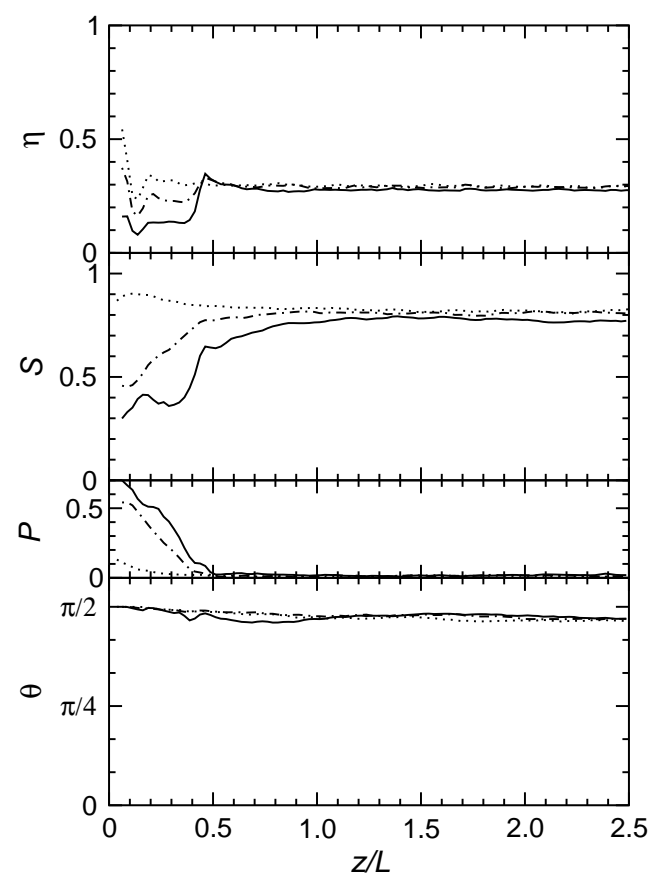

Fig. 1 - Profiles for $L_{\mathrm{ch}}=L / 3$. Surface coverage $\sigma=0 \%$ (dots), $3.75 \%$ (dash-dotted line), $10 \%$ (solid line).

$\sigma$ further produces dramatic effects, and we distinguish two regimes: tilted anchoring and homeotropic anchoring.

For surface chain coverages of $\sigma=2.5 \%-5 \%$, some tilting of the bulk phase by about $\pi / 6$ from the in-plane direction, $\theta \approx \pi / 3$ from the normal, is seen in fig. 2 . It is interesting that this is associated with an extended region $z<L$ of reduced order parameter $S$. Although the packing fraction within the grafting region is lower than in bulk, the variation is not dramatic.

For $\sigma=10 \%$, different behaviour is seen, which results in homeotropic anchoring. Packing fraction $\eta(z)$, order parameter $S(z)$, and director tilt angle $\theta(z)$, all change rapidly around $z=$ $(L+D) / 2$. The first peak in $\eta(z)$ corresponds to a layer of spherocylinders lying normal to the surface; this high-density region extends over a substantial range indicating interdigitation of bulk liquid-crystal molecules with the grafting region. A subsidiary peak can be seen at a distance $z \approx L_{\mathrm{ch}}+L / 2$ corresponding to normally oriented molecules lying above the chain ends.

For the full-length grafted rods $L_{\mathrm{ch}}=L$ the behaviour is somewhat different, as illustrated in fig. 3. At the lowest coverage, $\sigma=1.25 \%$ (not shown), effects are limited to a small peak in $\eta(z)$ at $z \approx L / 2$ and a reduction in $S(z)$ in the region $L / 2<z<L$, corresponding to an enhancement in the proportion of rods oriented perpendicular to the surface. However, this is not sufficient to affect the bulk orientation. Similar, but larger, effects in $\eta(z)$ and $S(z)$ are seen for $\sigma=2.5 \%$, as shown in fig. 3. Here, though, the extended region of low ordering is accompanied by a bend in the director within the grafting region, from $\theta=\pi / 2$ parallel to the surface, to a tilt of $\theta \approx \pi / 4$ at $z \approx L / 2$. In the range $L / 2<z<L$ an enhanced packing fraction $\eta(z)$ is seen, corresponding to a layer of molecules interdigitated with those of the surface chains.

Increasing the coverage of chains to $\sigma=3.75 \%$ enhances these effects and leads to homeotropic alignment of the liquid crystal perpendicular to the surface, as shown in fig. 3. Broad 


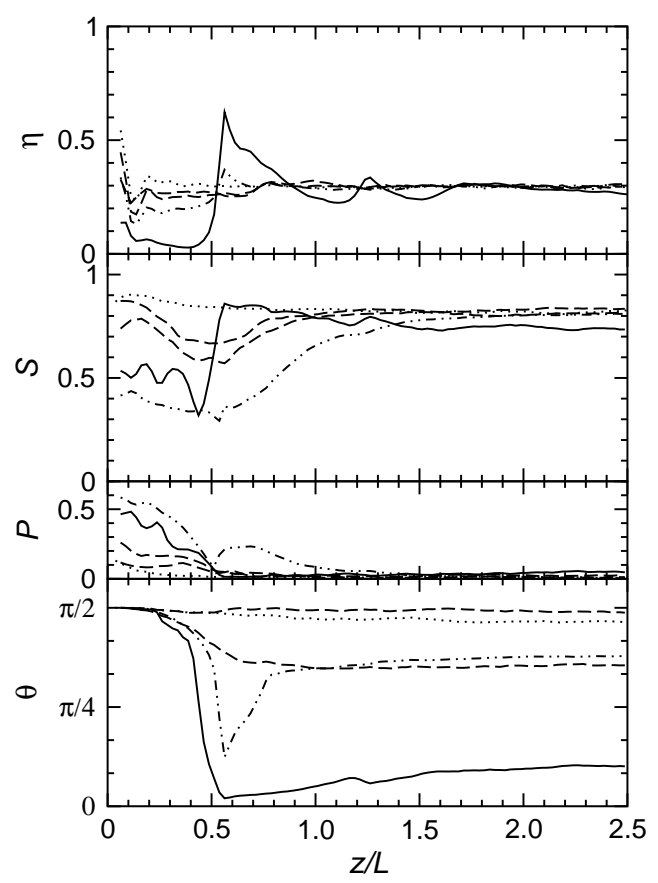

Fig. 2 - Profiles for $L_{\mathrm{ch}}=2 L / 3$. Surface coverage $\sigma=0 \%$ (dots), $2.5 \%$ (dashes; two sets of results shown), $5 \%$ (dash-double-dotted line), $10 \%$ (solid line).

subsidiary peaks at larger distances from the wall indicate further weak layering.

For a surface coverage of $\sigma=5 \%$ (see fig. 3) and $\sigma=10 \%$ (not shown, but similar), new behaviour is seen. The density near the surface is very much reduced, but there is a very high peak at $z \approx(L+D) / 2$ corresponding to a well-defined layer of normally oriented molecules, $\theta(z)=0$. However, there is a minimum in $\eta(z)$ at $z \approx L+D$; subsequently, the bulk nematic phase is oriented with $\theta(z)=\pi / 2$, parallel to the (rough) surface presented by this tightly packed layer.

Discussion and conclusions. - The effects of a number of different simple surfaces on the anchoring of a nematic liquid crystal have been investigated using computer simulation (see summary in table I). The results have been shown to be surprisingly rich for such a simple model; a number of different orientational effects dependent on both the density and attachment details of the surface chains have been observed.

A clear homeotropic alignment has been seen in three of the cases studied. The first occurs for chains with the same length as the liquid crystal, where the reorientation is due to a tendency for some molecules to lie parallel with the surface chains above a layer of molecules lying on the surface, these stretch into the bulk and promote its rotation; this effect disappears as the density of chains is increased. These profiles are similar to those seen in ref. [11] for a system of particles interacting via the hard Gaussian overlap potential. Second, for chains with a shorter length there is a "locking in" of the liquid crystal with a dense monolayer on the surface.

One unexpected finding has been that for some systems the liquid crystal sees a rough wall effectively above the ends of the chains, that aligns the sample in the $x y$ plane. Again this effect was observed for two different systems with differing structures in the region of 


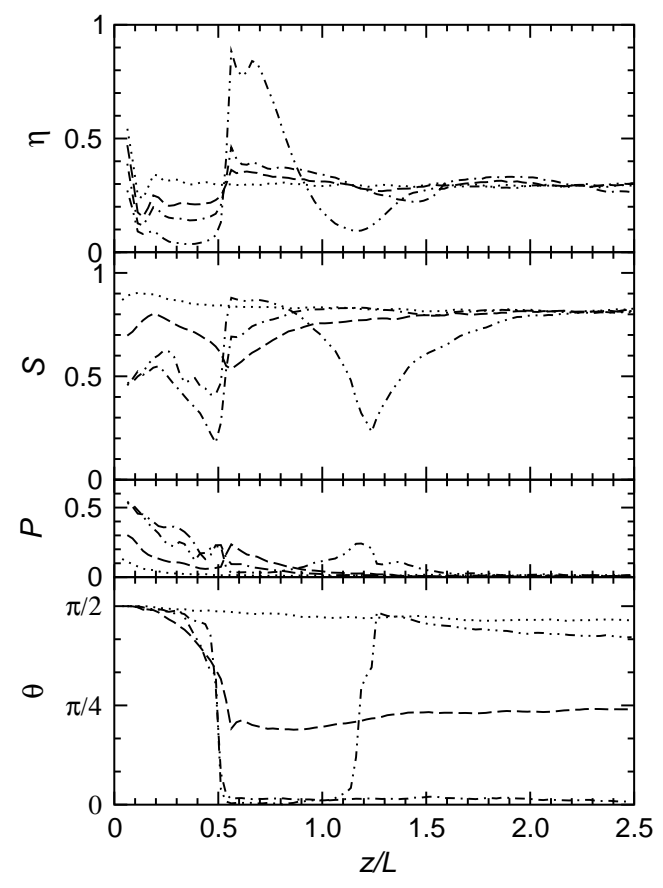

Fig. 3 - Profiles for $L_{\mathrm{ch}}=L$. Surface coverage $\sigma=0 \%$ (dots), $2.5 \%$ (dashes), $3.75 \%$ (dash-dotted line), $5 \%$ (dash-double-dotted line).

the chains. For chains with the same length as the bulk a smectic layer forms in the surface region, this layer is highly localised to the region above the substrate and the bulk liquid lies flat above this. Such effects have been seen previously in simulations of Gay-Berne units interacting with a surface that gives homeotropic alignment of the liquid crystal [12]; here though the transition occurs purely due to entropic reasons. Spherocylinders have been found to orient perpendicular to smectic layers in previous studies [13].

Finally, in a few cases the interplay between planar and homeotropic anchorings has led to tilted cell profiles. One method of creating such a profile has been considered for long polymer brushes by Halperin and Williams $[14,15]$ who have predicted a second-order transition to a tilted state with increased density of chains. Similar simulations of short polymer brushes have been performed by Lange and Schmid who have also seen tilted systems [16-18]. The distinguishing feature of the present work is that we have found tilted configurations for fixed inflexible chains that are of the same order of length as the liquid-crystalline molecules. Such tilting has been observed before for the case of molecules which have attractive interactions [19, 20]; this is the first time that tilt has been found in a purely entropic system.

TABLE I - Anchoring types for different surface coverages and grafted rod lengths.

\begin{tabular}{lccccc}
\hline$\sigma=$ & $1.25 \%$ & $2.5 \%$ & $3.75 \%$ & $5 \%$ & $10 \%$ \\
\hline$L_{\text {ch }}=\frac{1}{3} L$ & Planar & Planar & Planar & Planar & Planar \\
$L_{\text {ch }}=\frac{2}{3} L$ & Planar & Tilted/Planar & Tilted & Tilted & Homeotropic \\
$L_{\text {ch }}=L$ & Planar & Tilted & Homeotropic & Decoupled planar & Decoupled planar \\
\hline
\end{tabular}


The authors would like to thank F. Schmid and H. LANGE for useful discussions. MTD acknowledges the financial support of the EPSRC and Hewlett-Packard. The work presented was carried out at the University of Bristol.

\section{REFERENCES}

[1] Sluckin T. J. and Poniewierski A., Fluid Interfacial Phenomena, edited by Croxton C. A. (Wiley) 1986.

[2] Poniewierski A. and Samborski A., J. Chem. Phys., 105 (1996) 7632.

[3] Poniewierski A. and Samborski A., Liq. Cryst., 23 (1997) 377.

[4] Rodríguez-Ponce I., Romero-Enrique J. M., Velasco E., Mederos L. and Rull L. F., Phys. Rev. Lett., 82 (1999) 2697.

[5] Braun F. N., J. Chem. Phys., 111 (1999) 4851.

[6] JÉrôme B., Rep. Prog. Phys., 54 (1991) 391.

[7] Cognard J., Mol. Crys. Liq. Cryst. (Suppl. Ser.) A, 5 (1982) 1.

[8] Matsumoto S., Kawamoto M. and Kaneko N., Appl. Phys. Lett., 27 (1975) 268.

[9] Bolhuis P. and Frenkel D., J. Chem. Phys., 106 (1997) 666.

[10] Frenkel D. and Smit B., Understanding Molecular Simulation: From Algorithms to Applications (Academic Press, London) 1996.

[11] Cleaver D. J. and Teixeira P. I. C., Chem. Phys. Lett., 338 (2001) 1.

[12] Palermo V., Biscarini F. and Zannoni C., Phys. Rev. E, 57 (1998) 2519.

[13] van Roij R., Bolhuis P., Mulder B. and Frenkel D., Phys. Rev. E, 52 (1995) R1277.

[14] Halperin A. and Williams D. R. M., Europhys. Lett., 21 (1993) 575.

[15] Halperin A. and Williams D. R. M., J. Phys. Condens. Matter, 6 (1994) A297.

[16] Lange H. and Schmid F., Eur. Phys. J. E, 7 (2002) 175.

[17] Lange H. and Schmid F., Comput. Phys. Commun., 147 (2002) 276.

[18] Lange H. and Schmid F., J. Chem. Phys., 117 (2002) 362.

[19] Zhang Z., Chakrabarti A., Mouritsen O. G. and Zuckermann M. J., Phys. Rev. E, 53 (1996) 2461.

[20] Wall G. D. and Cleaver D. J., Phys. Rev. E, 56 (1997) 4306. 